\title{
Mother's prenatal and postpartum depression symptoms and infant's sleep problems at 6 months
}

\section{Cláudia Castro Dias () | Bárbara Figueiredo}

School of Psychology, University of Minho, Braga, Portugal

\section{Correspondence}

Cláudia Castro Dias, Universidade do Minho, Escola de Psicologia, Campus de Gualtar, 4710-057 Braga, Portugal.

Email:cdias@psi.uminho.pt

\section{Funding information}

This study was conducted at Psychology Research Centre (UID/PSI/01662/2013), University of Minho, and supported by the Portuguese Foundation for Science and Technology and the Portuguese Ministry of Education and Science through national funds and co-financed by FEDER through COMPETE2020 under the PT2020 Partnership Agreement, under the Grant No. POCI-01-0145-FEDER007653. This research was supported by the FEDER Funds through the Programa Operacional Factores de CompetitividadeCOMPETE and by National Funds through FCTFundação para a Ciência e a Tecnologia under the Grant No. PTDC/SAU/SAP/116738/2010 and by a PhD Grant No. SFRH/BD/113005/2015.

\begin{abstract}
Mother's prenatal and postpartum depression have been associated with infant's sleep problems. This study aimed to analyze (a) the effects of mother's prenatal and postpartum depression symptoms, including the effects of prenatal and postpartum anxiety and depression scores of the Edinburgh Postnatal Depression Scale (EPDS), on infant's sleep problems at 6 months, and (b) the interaction effect between mother's prenatal and postpartum depression symptoms and infant's sex on infant's sleep problems at 6 months. The sample was comprised of 164 mother-infant dyads whose mothers completed measures of depression at the third trimester of pregnancy, 2 weeks, 3 and 6 months postpartum and a measure of infant's sleep problems at 6 months (CSHQ-I). Mother's prenatal depression symptoms, specifically depression scores of the EPDS, predicted more infant's sleep anxiety and daytime sleepiness, while mother's depression symptoms at 2 weeks postpartum, specifically anxiety scores of the EPDS, predicted more bedtime resistance and CSHQ-I total scores at 6 months. Boys of mothers with more prenatal depression symptoms presented more sleep anxiety at 6 months. Both mother's prenatal and early postpartum depression symptoms have a negative effect on the emergence of infant's sleep problems. Additionally, boys seem more vulnerable to mother's prenatal depression symptoms.
\end{abstract}

\section{KEYWORDS}

development, postpartum depression, prenatal depression, sex differences, sleep problems
The developmental psychopathology conceptual model focuses on the study of the adaptative and maladaptive development (Sroufe, 2009, 2016). According to this conceptual model, infant development is affected by the accomplishment of different tasks across the life span (e.g., Rutter, 1996). Regarding sleep during infancy, literature suggested that four sleep developmental tasks are achieved during the first year of life, namely the ability of (a) spending more time awake than asleep, (b) concentrating sleep during the night, (c) self-soothing, and (d) sleeping through the night (Figueiredo, Dias, Pinto, \& Field, 2016; Galland, Taylor, Elder, \& Herbison, 2012; Goodlin-
Jones, Burnham, Gaylor, \& Anders, 2001). The accomplishment of these sleep developmental tasks may lead to infant adjustment and adequate development, while its failure may lead to the emergence of sleep problems (Byars, Yolton, Rausch, Lanphear, \& Beebe, 2012; Sette, Baumgartner, Ferri, \& Bruni, 2017). The developmental psychopathology conceptual model also highlights the importance of understanding the mechanisms through which development is affected and maladaptive trajectories are established (Rutter \& Sroufe, 2000; Sroufe, 2016).

Maternal depression is highly prevalent, with rates ranging 10-20\% during pregnancy (Couto et al., 2016; 


\section{Key Findings}

1. Key finding 1 :

Mother's prenatal depression scores of the EPDS predicted more infant's sleep anxiety and daytime sleepiness at 6 months.

2. Key finding 2:

Mother's anxiety scores of the EPDS predicted more infant's bedtime resistance and total sleep problems.

These findings show the importance of screening for mother's prenatal and early postpartum depression as part of routine perinatal care to identify infants at risk of presenting sleep problems. Moreover, considering that mother's prenatal and postpartum depression both affect infant's sleep problems, these results acknowledge the need of intervening on infant's sleep problems when developing mother's depression interventions.

3. Key finding 3 :

Boys of mothers with more prenatal depression symptoms presented more sleep anxiety at 6 months postpartum. More research is needed to explore the differential effect of mother's depression symptoms on infant's sleep problems according to infant's sex.

Figueiredo \& Conde, 2011a; Underwood, Waldie, D’Souza, Peterson, \& Morton, 2016; Verreault et al., 2014), and 13-16.4\% during the postpartum period (Figueiredo \& Conde, 2011a; Underwood et al., 2016; Verreault et al., 2014). Mother's prenatal (Field et al., 2007; Gerardin et al., 2011; Nevarez, Rifas-Shiman, Kleinman, Gillman, \& Taveras, 2010) and postpartum depression (Bayer, Hiscock, Hampton, \& Wake, 2007; Gress-Smith, Luecken, LemeryChalfant, \& Howe, 2012; Hughes, Gallagher, \& Hannigan, 2015) were both associated with infant's sleep problems. However, studies on the effects of maternal depression on infant's sleep problems did not analyze the concurrent effect of both maternal prenatal and postpartum depression.

Maternal depression effects on infant development may occur through different potential mechanisms: genetic factors, epigenetic change, gene-environment interaction, dysfunctional biological mechanisms, maladaptive cognitions or problematic mother-infant interactions (Field, 2010; Glover, O'Donnell, O'Connor, \& Fisher, 2018; Stein et al., 2014). Potential moderators of the effects of maternal depression on infant development were also iden-

\section{Statement of relevance to the field of infant and early childhood mental health}

These results extend the knowledge on infant's sleep research, by suggesting that (a) both mother's prenatal and postpartum depression symptoms may have a negative effect on infant's sleep problems at 6 months, and (b) the impact of mother's prenatal depression symptoms on infant's sleep anxiety may be different on boys and girls.

tified: infant's characteristics, namely sex and temperament, and maternal socioeconomic status and parenting style (Beeghly et al., 2017; McGinnis, Bocknek, Beeghly, Rosenblum, \& Muzik, 2015; Stein et al., 2014). The differential susceptibility theory argues that infants are not equally affected by the environmental influences, either positively or negatively (Belsky, Bakermans-Kranenburg, \& Van I. Jzendoorn, 2007). Regarding infant's characteristics, research has shown that boys present more dysregulated sleep patterns than girls in the presence of mother's prenatal depression (Netsi et al., 2015). These differences may be related to the sex differences already present during the fetal development (Rosenfeld, 2015; Sutherland \& Brunwasser, 2018). Namely, male infants were shown to present more difficulty to adapt to embryotic adverse environments, for example, having a mother with prenatal depression symptoms (Rosenfeld, 2015; Sutherland \& Brunwasser, 2018). Thus, when exposed to prenatal adverse environment, male infants were shown to present more pervasive effects on their further development than females (DiPietro \& Voegtline, 2017; Sandman, Glynn, \& Davis, 2013; Sutherland \& Brunwasser, 2018).

Both mother's prenatal and postpartum depression symptoms were found to negatively affect infant development (Gerardin et al., 2011; Gress-Smith et al., 2012; Hughes et al., 2015). Additionally, previous literature suggested that infant's sex moderates the effect of maternal depression on infant development (Beeghly et al., 2017; McGinnis et al., 2015; Stein et al., 2014). Considering these previous findings, this longitudinal study aimed to analyze, under the context of developmental psychopathology conceptual model, (a) the effects of mother's prenatal and postpartum depression symptoms, including the effects of prenatal and postpartum anxiety and depression scores of the EPDS, on infant's sleep problems at 6 months, and (b) the interaction effect between mother's prenatal and postpartum depression symptoms and infant's sex on infant's sleep problems at 6 months. 
TABLE 1 Sociodemographic characteristics

\begin{tabular}{|c|c|c|c|}
\hline & & & $\frac{\text { Total }}{\frac{N=164}{\%}}$ \\
\hline \multirow[t]{11}{*}{ Mother } & \multirow[t]{3}{*}{ Age } & $18-24$ & 9.1 \\
\hline & & $25-34$ & 73.8 \\
\hline & & $35-44$ & 17.1 \\
\hline & \multirow[t]{3}{*}{ Socioeconomic level } & High & 53.2 \\
\hline & & Medium & 39.7 \\
\hline & & Low & 7.1 \\
\hline & Marital status & Married/cohabiting & 82.2 \\
\hline & \multirow[t]{2}{*}{ Years of schooling } & $\leq 12$ & 33.7 \\
\hline & & $>12$ & 66.3 \\
\hline & \multirow[t]{2}{*}{ Gestational age } & $<37$ weeks & 1.8 \\
\hline & & $\geq 37$ weeks & 98.2 \\
\hline \multirow[t]{4}{*}{ Gestation } & \multirow[t]{2}{*}{ Resuscitation at birth } & No & 92.1 \\
\hline & & Yes & 7.9 \\
\hline & \multirow[t]{2}{*}{ Type of delivery } & Vaginal & 66.5 \\
\hline & & Caesarean & 33.5 \\
\hline \multirow[t]{2}{*}{ Infant } & Sex & Female & 46.3 \\
\hline & First born & No & 10.4 \\
\hline
\end{tabular}

\section{1 | METHODS}

\section{1 | Participants}

The sample was comprised of 164 mother-infant dyads whose mothers completed measures of depression at the third trimester of pregnancy, 2 weeks, 3 and 6 months postpartum, and a measure of infant's sleep problems at 6 months. The majority of the mothers were white (93.7\%), married or cohabiting (82.2\%), employed (76.8\%), and more than half were aged 25-34 years old (73.8\%), belonged to a high socioeconomic level (53.2\%), and had more than 12 years of schooling (66.3\%). The majority of infants were born at term ( $\geq 37$ weeks of gestation; $98.2 \%$ ), were not resuscitated at birth (92.1\%), had normal birth weight ( $\geq 2500 \mathrm{~g})(98.8 \%)$, and length ( $\geq 48 \mathrm{~cm} ; 79.3 \%)$, and were first born (89.6\%). More than half of the infants were born by vaginal delivery (66.5\%) and were males (53.7\%; Table 1).

\section{2 | Procedures}

This study took part from a larger longitudinal study that received approval of the Ethical Commissions of all the institutions involved. At the third trimester of pregnancy, 536 women who met the inclusion criteria were contacted at two public hospitals in the Northern Portugal, informed about the study's purposes and procedures and invited to participate. Women not reading/writing Portuguese, with gestational complications, psychiatric medication intake, and multiple births were excluded. Four hundred and eighty-five women (90.5\%) agreed to participate, signed an informed consent and completed measures of 
sociodemographic characteristics and depression symptoms at the third trimester of pregnancy. Women were contacted again and 317 (65.3\%) completed online measures of sociodemographic characteristics and depression symptoms at 2 weeks and $303(62.5 \%)$ at 3 months postpartum. From these women, 164 (54.1\%) completed measures of depression and infant's sleep problems at 6 months and were included in the study. Significant associations were found between participants who completed all the measures and those who did not complete all the measures, on parity, Wald $=6.72, \beta=-.94$, OR $=.39, p=.010$. Primiparous women were more likely to complete all the study measures.

\section{3 | Measures}

\subsection{1 | Sociodemographic characteristics}

The Sociodemographic Questionnaire is an instrument that assesses maternal demographic and obstetric information, and infants' sociodemographic and biometric information (e.g., age, marital status, occupational status, socioeconomic status, years of schooling, type of delivery, weeks of gestation).

\subsection{2 | Mother's depression symptoms}

The EPDS (Cox, Holden, \& Sagovsky, 1987) is a self-report measure, comprised of 10 items that are scored on a fourpoint Likert-type scale to assess pregnancy and postpartum depression symptoms within the previous 7 days (total scores ranging from 0 to 30). Although the Portuguese version validation used the EPDS as a unidimensional measure (Tendais, Costa, Conde, \& Figueiredo, 2014), different studies worldwide have suggested the EPDS to be comprised of two factors: the anxiety subscale and the depression subscale (e.g., Jomeen \& Martin, 2005; Matthey, 2008; Phillips, Charles, Sharpe, \& Matthey, 2009). Although some variation, several studies have shown the anxiety subscale to be comprised of three items (items 3, 4, and 5: EPDS-3A; Matthey, 2008; Phillips et al., 2009; SmithNielsen, Matthey, Lange, \& Væver, 2018), and the depression subscale to be comprised of the remaining seven items (Matthey, 2008; Phillips et al., 2009). Good internal consistency was found in the Portuguese version of the EPDS both during pregnancy and postpartum $(\alpha=.82$ and .88 ; Tendais et al., 2014). In the present sample, the EPDS total score showed good internal consistency at the third trimester of pregnancy $(\alpha=.85)$, and at 2 weeks, 3 and 6 months postpartum ( $\alpha$ s ranging from .82 to .89 ). Additionally, adequate to good internal consistency was found in the two subscales at the third trimester of pregnancy, 2 weeks, 3 and 6 months postpartum, with $\alpha$ s ranging from .68 to .82 in the anxiety subscale and $\alpha$ s ranging from .74 to .84 in the depression subscale.

\subsection{3 | Infant's sleep problems}

The CSHQ-I (Dias, Figueiredo, \& Pinto, 2018) was designed to assess sleep problems in infants aged 0 and 12 months old. It is a Portuguese adapted version of the Children's Sleep Habits Questionnaire-CSHQ (Owens, Spirito, \& McGuinn, 2000), a measure that has been used worldwide to assess sleep problems in preschool and school aged children (e.g., Goodlin-Jones, Sitnick, Tang, Liu, \& Anders, 2008; Owens et al., 2000; Waumans et al., 2010). The CSHQ-I is a retrospective questionnaire, comprised of 33 items scored in a three-point Likert-type scale that identifies infant's sleep problems based on the International Classification of Sleep Disorders (ICSD-1). It is comprised of four subscales: (a) Bedtime Resistance: infant's resistance to go to bed and frequent night waking (e.g., "Child falls asleep alone in own bed"); (b) Sleep Anxiety: infant's anxiety related to sleep, and items of sleepdisordered breathing (e.g., "Child awakens during night screaming, sweating, and inconsolable"); (c) Positive Sleep Habits: infant's bedtime and morning waking routines (e.g., "Child goes to bed at the same time at night"); and (d) Daytime Sleepiness: daytime consequences from sleep problems (e.g., "Child takes a long time to become alert in the morning"). The scores of the CSHQ-I total scale and subscales are obtained through the sum of the items divided by the number of items (scores ranging from 0 to 2). Higher scores in the CSHQ-I are indicative of more infant's sleep problems. Adequate to good internal consistency was found for the total scale $(\alpha=.77)$ and the subscales ( $\alpha$ s ranging from .52 and .77; Dias et al., 2018). In the present sample, the CSHQ-I total score presented adequate to good internal consistency for the total scale $(\alpha=.80)$ and subscales ( $\alpha$ s ranging from .36 and .78).

\subsection{Statistical analyses}

Statistical analyses were performed with SPSS version 22.0 (SPSS Inc., USA). Multiple linear regressions (stepwise method) were used to determine the stronger predictor (mother's prenatal or postpartum depression symptoms) of infant's sleep problems at 6 months. Models included mother's prenatal depression symptoms (EPDS total scores at the third trimester of pregnancy) and mother's postpartum depression symptoms (EPDS total scores at 2 weeks, 3 months and 6 months) as independent variables (IVs) 
and infant's CSHQ-I total score, bedtime resistance, sleep anxiety, positive sleep habits, and daytime sleepiness at 6 months as dependent variables (DVs).

To analyze the effects of prenatal and postpartum anxiety and depression scores of the EPDS on infant's sleep problems at 6 months, further multiple linear regressions (stepwise method) were performed to determine the stronger predictor (the prenatal and postpartum anxiety and depression scores of the EPDS) of infant's sleep problems at 6 months. Models included mother's prenatal depression and anxiety symptoms (anxiety and depression subscales of EPDS at the third trimester of pregnancy) and mother's postpartum depression and anxiety symptoms (anxiety and depression subscales of EPDS at 2 weeks, 3 months and 6 months) as independent variables (IVs) and infant's CSHQ-I total score, bedtime resistance, sleep anxiety, positive sleep habits, and daytime sleepiness at 6 months as dependent variables (DVs).

To analyze the interaction effect between mother's prenatal and postpartum depression symptoms and infant's sex on infant's sleep problems at 6 months, moderation models were tested according to Baron and Kenny guidelines (1986). Models to analyze the interaction effect between mother's prenatal depression symptoms and infant's sex $(-1=$ male; $1=$ female $)$ on infant's sleep anxiety, and daytime sleepiness included: (a) mother's prenatal depression symptoms (EPDS total scores at the third trimester of pregnancy) as IV (path a); (b) infant's sex as IV (path b); and (c) the interaction between mother's prenatal depression symptoms and infant's sex as IV (path c). Infant's sleep anxiety, and daytime sleepiness at 6 months were included as DVs. Models to analyze the interaction effect between mother's postpartum depression symptoms and infant's sex on infant's CSHQ-I total score and bedtime resistance included: (a) mother's depression symptoms at 2 weeks (EPDS total scores at 2 weeks postpartum) as IV (path a); (b) infant's sex as IV (path b); and (c) the interaction between mother's depression symptoms at 2 weeks postpartum and infant's sex as IV (path c). Infant's CSHQ-I total score and bedtime resistance at 6 months were included as DVs. Further moderation models were performed to analyze the interaction effect between prenatal and postpartum anxiety and depression scores of the EPDS and infant's sex on infant sleep problems at 6 months. The same models were tested according to Baron and Kenny guidelines (1986). The first models included: (a) prenatal depression scores of the EPDS as IV (path a); (b) infant's sex as IV (path b); and (c) the interaction between prenatal depression scores of the EPDS and infant's sex as IV (path c). Infant's sleep anxiety, and daytime sleepiness at 6 months were included as DVs. The second models included: (a) anxiety scores of the EPDS at 2 weeks as IV (path a); (b) infant's sex as IV (path b); and (c) the interaction between anxiety scores of the EPDS at 2 weeks postpartum and infant's sex as IV (path c). Infant's CSHQI total score and bedtime resistance at 6 months were included as DVs.

\section{RESULTS}

\section{1 | Preliminary analyses}

Means and standard deviations for mother's prenatal and postpartum depression symptoms and infant's sleep problems at 6 months are presented in Table 2. The scores of mother's prenatal and postpartum depression symptoms reported in the present study are similar to the scores found in previous studies with different Portuguese samples (e.g., Figueiredo \& Conde, 2011b). A previous study reported mean scores of depression symptoms of $5.97(\mathrm{SD}=4.05)$ at the third trimester of pregnancy (e.g., Figueiredo \& Conde, 2011b), similar to the 6.95 (SD = 4.29) found in the present sample. Additionally, mean scores of depression symptoms of $5.88(\mathrm{SD}=4.64)$ and $4.69(\mathrm{SD}=4.13)$ were reported at childbirth and at 3 months postpartum in a previous study (Figueiredo \& Conde, 2011b), similar to the 5.35 $(\mathrm{SD}=4.44$ ) and $4.54(\mathrm{SD}=3.59)$ found in the present sample at 2 weeks and 3 months postpartum.

Correlations between mother's prenatal, postpartum depression symptoms, and infant's sleep problems at 6 months are also presented in Table 2. Nonsignificant correlations were found between mother's prenatal and postpartum depression symptoms and sociodemographic variables (maternal age, ethnicity, socioeconomic level, marital status, occupational status, gestational age, type of delivery, resuscitation at birth, infant's sex, birth weight, birth length, birth order). A significant correlation was found between mother's postpartum depression symptoms at 3 months postpartum and maternal years of schooling, $r$ $=.166, p=.034$. Considering this result, regression models were adjusted for maternal years of schooling.

\section{2 | Mother's prenatal and postpartum depression symptoms (EPDS total score) as predictors of infant's sleep problems at 6 months postpartum}

Mother's prenatal and postpartum depression symptoms (EPDS total score) were tested as predictors of infant's sleep problems at 6 months (stepwise regression method). Five models were conducted: one for the CSHQ-I total score, and one for each CSHQ-I subscale (bedtime resistance, sleep anxiety, positive sleep habits, and daytime sleepiness) (Table 3). 
TA B L E 2 Study's variables' means, standard deviations, and correlations

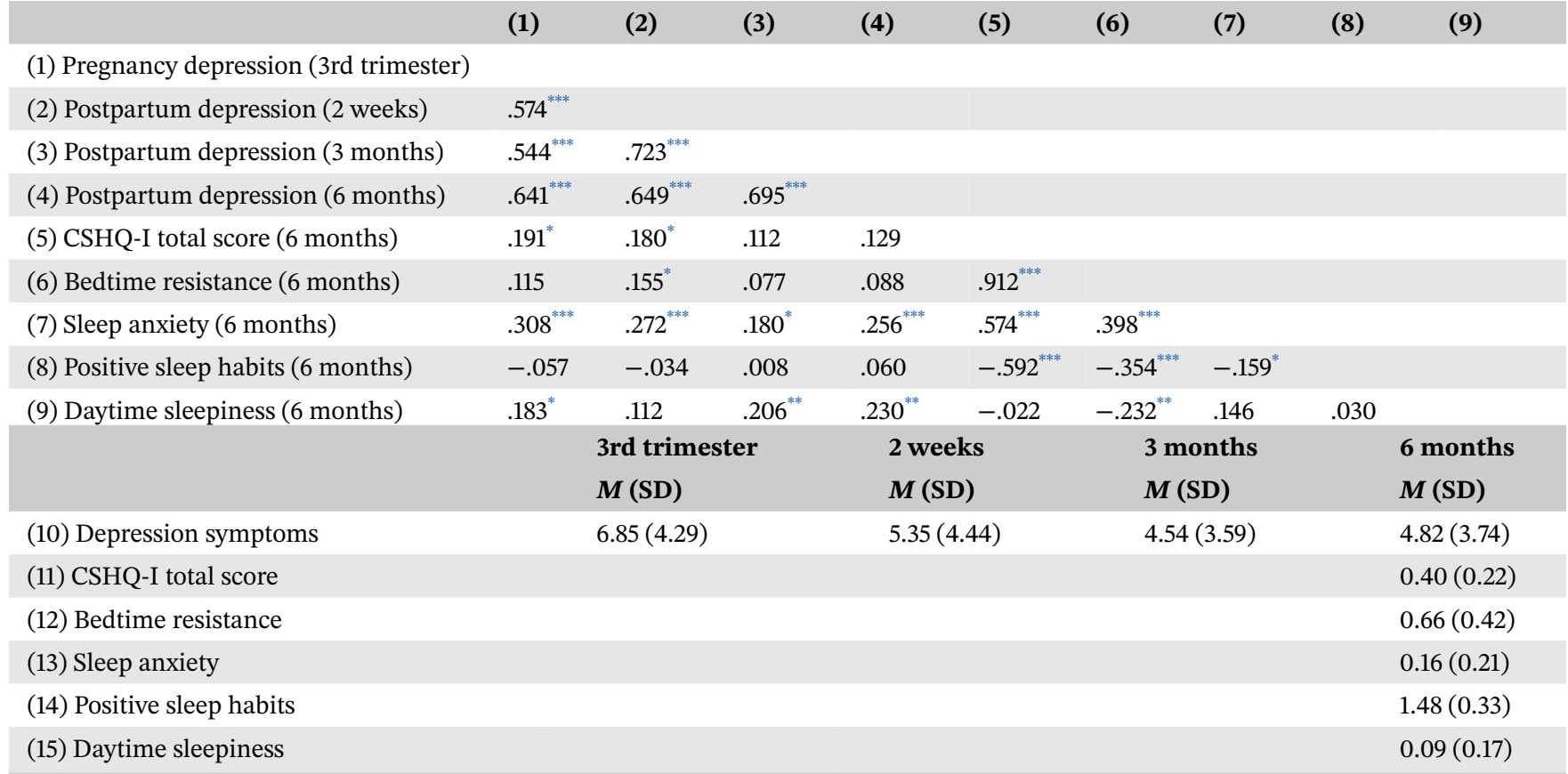

Abbreviations: $M$, mean; SD, standard deviation.

${ }^{*} p<.05 .{ }^{* *} p<.01 .{ }^{* * *} p<.001$.

TA B LE 3 Prenatal and postpartum depression symptoms as predictors of infant's sleep problems at 6 months

\begin{tabular}{|c|c|c|c|c|}
\hline & $R^{2}\left(R^{2}{ }_{A j}\right)$ & $\boldsymbol{F}$ & $\boldsymbol{B}$ & $t$ \\
\hline \multicolumn{5}{|l|}{ Model 1} \\
\hline \multicolumn{5}{|l|}{ CSHQ-I total score } \\
\hline \multicolumn{5}{|l|}{ Model 2} \\
\hline \multicolumn{5}{|l|}{ Bedtime resistance } \\
\hline \multicolumn{5}{|l|}{ Sleep Anxiety } \\
\hline Pregnancy depression symptoms (3rd trimester) & $0.15(0.14)$ & $13.96^{* * *}$ & 0.35 & $4.77^{* * *}$ \\
\hline \multicolumn{5}{|l|}{ Model 5} \\
\hline \multicolumn{5}{|l|}{ Daytime Sleepiness } \\
\hline
\end{tabular}

Note. Models were adjusted for maternal years of schooling.

${ }^{*} p<.05 .{ }^{* *} p<.01 .{ }^{* * *} p<.001$.

The regression models explained between $4 \%$ and $15 \%$ of the variance (adjusted $R^{2}$ range $=.03-.14$ ). Mother's prenatal depression symptoms (EPDS total score) were found to be the strongest predictor of infant's sleep anxiety $(\beta=$ $0.35, p<.001)$ and daytime sleepiness $(\beta=0.21, p=.001)$. Mother's postpartum depression symptoms (EPDS total score) at 2 weeks were found to be the strongest predictor of infant's CSHQ-I total score $(\beta=0.25, p=.002)$ and bedtime resistance $(\beta=0.20, p=.014)$ (see Table 3$)$. Mother's prenatal and postpartum depression symptoms (EPDS total score) did not predict infant's positive sleep habits at 6 months.

\subsection{Prenatal and postpartum anxiety and depression scores of the EPDS as predictors of infant's sleep problems at 6 months postpartum}

Further analyses were performed with the prenatal and postpartum anxiety and depression scores of the EPDS. The regression models explained between 4 and $12 \%$ of the variance (adjusted $R^{2}$ range $=.06-.13$ ). The depression scores of the EPDS at the third trimester of pregnancy were found to be the strongest predictor of infant's sleep anxiety 
TA B LE 4 Prenatal and postpartum depression symptoms (anxiety and depression scores) as predictors of infant's sleep problems at 6 months

\begin{tabular}{|c|c|c|c|c|}
\hline & $R^{2}\left(R^{2}{ }_{A j}\right)$ & $\boldsymbol{F}$ & $\boldsymbol{B}$ & $t$ \\
\hline \multicolumn{5}{|l|}{ Model 1} \\
\hline \multicolumn{5}{|l|}{ CSHQ-I total score } \\
\hline \multicolumn{5}{|l|}{ Model 2} \\
\hline \multicolumn{5}{|l|}{ Bedtime resistance } \\
\hline \multicolumn{5}{|l|}{ Model 3} \\
\hline \multicolumn{5}{|l|}{ Sleep anxiety } \\
\hline Pregnancy depression scores (3rd trimester) & $0.13(0.12)$ & $12.09^{* * *}$ & 0.32 & $4.38^{* * *}$ \\
\hline \multicolumn{5}{|l|}{ Model 5} \\
\hline \multicolumn{5}{|l|}{ Daytime sleepiness } \\
\hline
\end{tabular}

Note. Models were adjusted for maternal years of schooling.

${ }^{*} p<.05 .{ }^{* *} p<.01 .{ }^{* * *} p<.001$.

$(\beta=0.32, p<.001)$, and daytime sleepiness $(\beta=0.23, p$ $=.003)$ at 6 months. The anxiety scores of the EPDS at 2 weeks postpartum were found to be the strongest predictor of infant's CSHQ-I total score $(\beta=0.29, p<.000)$ and bedtime resistance $(\beta=0.25, p=.002)$ at 6 months (see Table 4). Prenatal and postpartum anxiety and depression scores of the EPDS did not predict infant's positive sleep habits at 6 months.

\section{$2.4 \quad$ The interaction effect between mother's prenatal and postpartum depression symptoms (EPDS total scores) and infant's sex on infant's sleep problems at 6 months}

The interaction effect between mother's prenatal and postpartum depression symptoms (EPDS total scores) and infant's sex on infant's sleep problems at 6 months was also tested. Considering the results found in the linear regression analyses, it was tested (a) the interaction effect between mother's prenatal depression symptoms (EPDS total score) and infant's sex on infant's sleep anxiety, and daytime sleepiness at 6 months; (b) the interaction effect between mother's depression symptoms (EPDS total score) at 2 weeks and infant's sex on infant's CSHQ-I total score and bedtime resistance at 6 months; (c) the interaction effect between mother's prenatal depression scores of the EPDS and infant's sex on infant's sleep anxiety, and daytime sleepiness at 6 months; and (d) the interaction effect between and mother's anxiety scores of the EPDS at 2 weeks postpartum and infant's sex on infant's CSHQ-I total score and bedtime resistance at 6 months.

\subsection{1 | The interaction effect between mother's prenatal depression symptoms (EPDS total score) and infant's sex on infant's sleep anxiety, and daytime sleepiness at 6 months}

First linear regressions (paths a) explained between 4.3 and $12 \%$ of variance (adjusted $R^{2}$ range $=.04-.12$ ) and revealed that mother's depression symptoms (EPDS total score) at the third trimester of pregnancy predicted infant's sleep anxiety $(\beta=0.35, p<.001)$, and daytime sleepiness $(\beta$ $=0.21, p=.007)$ at 6 months postpartum. Second linear regressions (paths b) explained between 4.4 and $12 \%$ of variance (adjusted $R^{2}$ range $=.03-.12$ ) and revealed that infant's sex did not predict infant's sleep anxiety $(\beta=$ $-0.02, p=.825)$, and daytime sleepiness $(\beta=-0.08, p=$ .310 ). Third linear regressions (paths c) explained between 6.5 and $14 \%$ of variance (adjusted $R^{2}$ range $=.05-.13$ ) and revealed that the interaction between mother's depression symptoms at the third trimester of pregnancy (EPDS total score) and infant's sex significantly predicted infant's sleep anxiety $(\beta=-0.28, p=.047)$ at 6 months, but did not significantly predict infant's daytime sleepiness $(\beta=-0.23$, $p=.112$ ) (see Table 5). The tested models revealed that the interaction between mother's depression symptoms at the third trimester of pregnancy (EPDS total score) and infant's sex significantly predicted infant's sleep anxiety at 6 months (see Figure 1). Boys of mothers with more depression symptoms during the third trimester of pregnancy presented more sleep anxiety at 6 months. No problems of multicollinearity were found between the variables of the regression analyses (Variance Inflation Factor was $<5$ and Tolerance $>.2$ ). 
TA B L E 5 The interaction effect between mother's prenatal and postpartum depression symptoms and infant's sex on infant sleep problems at 6 months

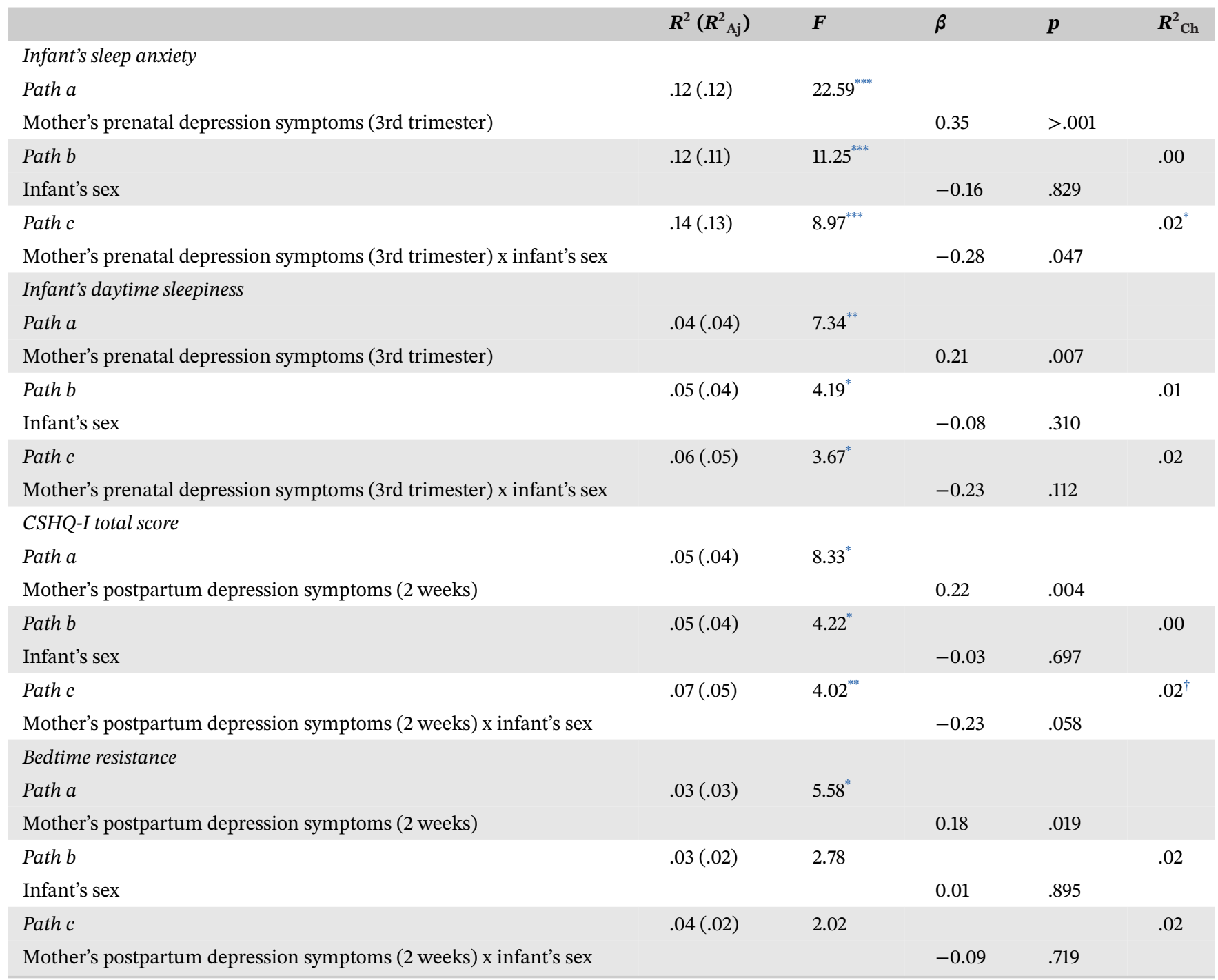

Note. $R_{\mathrm{Aj}}^{2}=$ adjusted $R^{2} ; R_{\mathrm{Ch}}^{2}=R^{2}$ change.

${ }^{\dagger} p>0.05<0.10 .{ }^{*} p<.05 .{ }^{* *} p<.01 .{ }^{* * *} p<.001$.

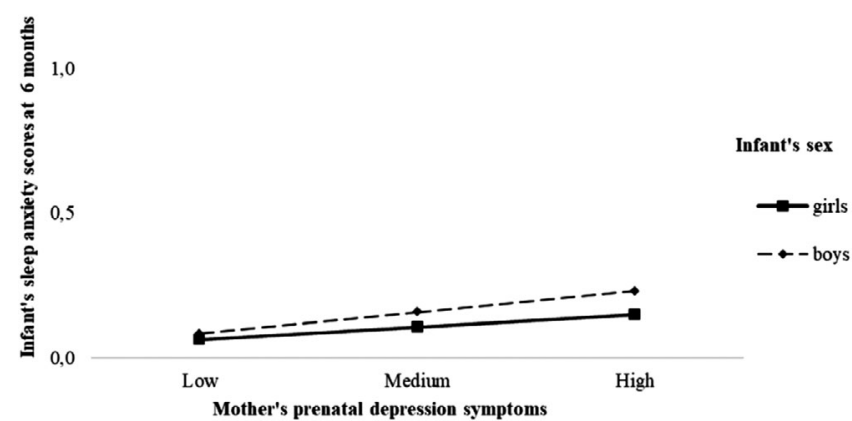

FIGURE 1 Interaction effect between mother's prenatal depression symptoms and infant's sex on infant's sleep anxiety at 6 months

\subsection{2 | The interaction effect between} mother's depression symptoms at 2 weeks (EPDS total score) and infant's sex on infant's CSHQ-I total score and bedtime resistance at 6 months

First linear regressions (path a) explained 3.3 and $4.9 \%$ of variance (adjusted $R^{2}$ range $=.03-.04$ ) and revealed that mother's depression symptoms at 2 weeks postpartum (EPDS total score) predicted infant's CSHQ-I total score $(\beta=0.22, p=.004)$, and bedtime resistance $(\beta=0.18, p=$ .019). Second linear regressions (path $b$ ) explained between 3.3 and $5.0 \%$ of variance (adjusted $R^{2}$ range $=.02-.04$ ) and revealed that infant's sex did not predict infant's CSHQI total score $(\beta=-0.03, p=.697)$, and bedtime resistance $(\beta=0.01, p=.895)$. Third linear regressions (path 
c) explained between 3.7 and $7.1 \%$ of variance (adjusted $R^{2}$ range $=.02-.05)$ and revealed that the interaction between mother's depression symptoms at 2 weeks postpartum (EPDS total score) and infant's sex did not significantly predict infant's CSHQ-I total score $(\beta=-0.23, p=.058)$, and bedtime resistance $(\beta=-0.09, p=.719)$ (see Table 5$)$. No problems of multicollinearity were found between the variables of the regression analyses (Variance Inflation Factor was $<5$ and Tolerance $>$.2).

\subsection{3 | The interaction effect between prenatal depression scores of the EPDS and infant's sex on infant's sleep anxiety, and daytime sleepiness at 6 months}

First linear regressions (paths a) explained between 5 and $10 \%$ of variance (adjusted $R^{2}$ range $=.05-.09$ ) and revealed that depression scores of the EPDS at the third trimester of pregnancy predicted infant's sleep anxiety $(\beta=0.31, p$ $<.001)$, and daytime sleepiness $(\beta=0.23, p=.003)$ at 6 months. Second linear regressions (paths $b$ ) explained between 6 and $10 \%$ of variance (adjusted $R^{2}$ range $=.05-$ .09 ) and revealed that infant's sex did not predict infant's sleep anxiety $(\beta=-0.03, p=.724)$, and daytime sleepiness $(\beta=-0.08, p=.286)$. Third linear regressions (paths c) explained between 8 and $12 \%$ of variance (adjusted $R^{2}$ range $=.06-.10)$ and revealed that the interaction between depression scores of the EPDS at the third trimester of pregnancy and infant's sex significantly predicted infant's sleep anxiety $(\beta=-0.22, p=.048)$ at 6 months, and did not significantly predict infant's daytime sleepiness $(\beta=-0.21$, $p=.072$ ) (see Table 6). The tested models revealed that the interaction between depression scores of the EPDS at the third trimester of pregnancy and infant's sex significantly predicted infant's sleep anxiety at 6 months. Boys of mothers with higher scores on the depression scores of the EPDS during the third trimester of pregnancy presented more sleep anxiety at 6 months postpartum. No problems of multicollinearity were found between the variables of the regression analyses (Variance Inflation Factor was $<5$ and Tolerance $>.2$ ).

\subsubsection{The interaction effect between anxiety scores of the EPDS at 2 weeks postpartum and infant's sex on infant's CSHQ-I total score and bedtime resistance at 6 months}

First linear regressions (path a) explained 5.7 and $7.8 \%$ of variance (adjusted $R^{2}$ range $=.05-.07$ ) and revealed that anxiety scores of the EPDS at 2 weeks predicted infant's
CSHQ-I total score $(\beta=0.28, p<.001)$, and bedtime resistance $(\beta=0.24, p=.002)$. Second linear regressions (path b) explained between 5.8 and $7.6 \%$ of variance (adjusted $R^{2}$ range $\left.=.05-.06\right)$ and revealed that infant's sex did not predict infant's CSHQ-I total score $(\beta=-0.01, p=.866)$, and bedtime resistance $(\beta=0.03, p=.764)$. Third linear regressions (path c) explained between 6.2 and $9.6 \%$ of variance (adjusted $R^{2}$ range $=.04-.08$ ) and revealed that the interaction between anxiety scores of the EPDS at 2 weeks postpartum and infant's sex did not significantly predict infant's CSHQ-I total score $(\beta=-0.24, p=.059)$, and bedtime resistance $(\beta=-0.10, p=.431)$ (see Table 5$)$. No problems of multicollinearity were found between the variables of the regression analyses (Variance Inflation Factor was $<5$ and Tolerance $>.2$ ).

\section{3 | DISCUSSION}

The findings of this study showed that mother's depression symptoms at the third trimester of pregnancy predicted more infant's sleep anxiety and daytime sleepiness at 6 months, while mother's depression symptoms at 2 weeks postpartum predicted more infant's bedtime resistance and total sleep problems at 6 months. The depression scores of the EPDS at the third trimester of pregnancy predicted more infant's sleep anxiety and daytime sleepiness at 6 months, while the anxiety scores of EPDS at 2 weeks postpartum predicted more infant's bedtime resistance and total sleep problems at 6 months. Results also showed that boys of mothers with more depression symptoms at the third trimester of pregnancy presented more sleep anxiety at 6 months.

Mother's depression symptoms at the third trimester of pregnancy (EPDS total score) were found to predict more infant's sleep problems at 6 months, specifically more sleep anxiety and daytime sleepiness. These results are in line with previous studies showing mother's prenatal depression is associated with the emergence of infant's sleep problems (Field et al., 2007; Gerardin et al., 2011; Nevarez et al., 2010). When further analyses were performed with the separated anxiety and depression scores of the EPDS, mother's depression scores at the third trimester of pregnancy significantly predicted more sleep anxiety and daytime sleepiness at 6 months. This result reinforced the major role of mother's prenatal depression symptoms on the emergence of further infant's sleep problems (Field et al., 2007; Gerardin et al., 2011; Nevarez et al., 2010). Also, this study adds to previous research on the adverse effects of mother's prenatal depression on infant development (e.g., Figueiredo, Pinto, Pacheco, \& Field, 2017; Gentile, 2017; Stein et al., 2014), by reporting the adverse effects on infant's sleep problems. In their turn, infant's sleep 
TA B L E 6 The interaction effect between mother's prenatal and postpartum depression symptoms (anxiety and depression scores) and infant's sex on infant sleep problems at 6 months

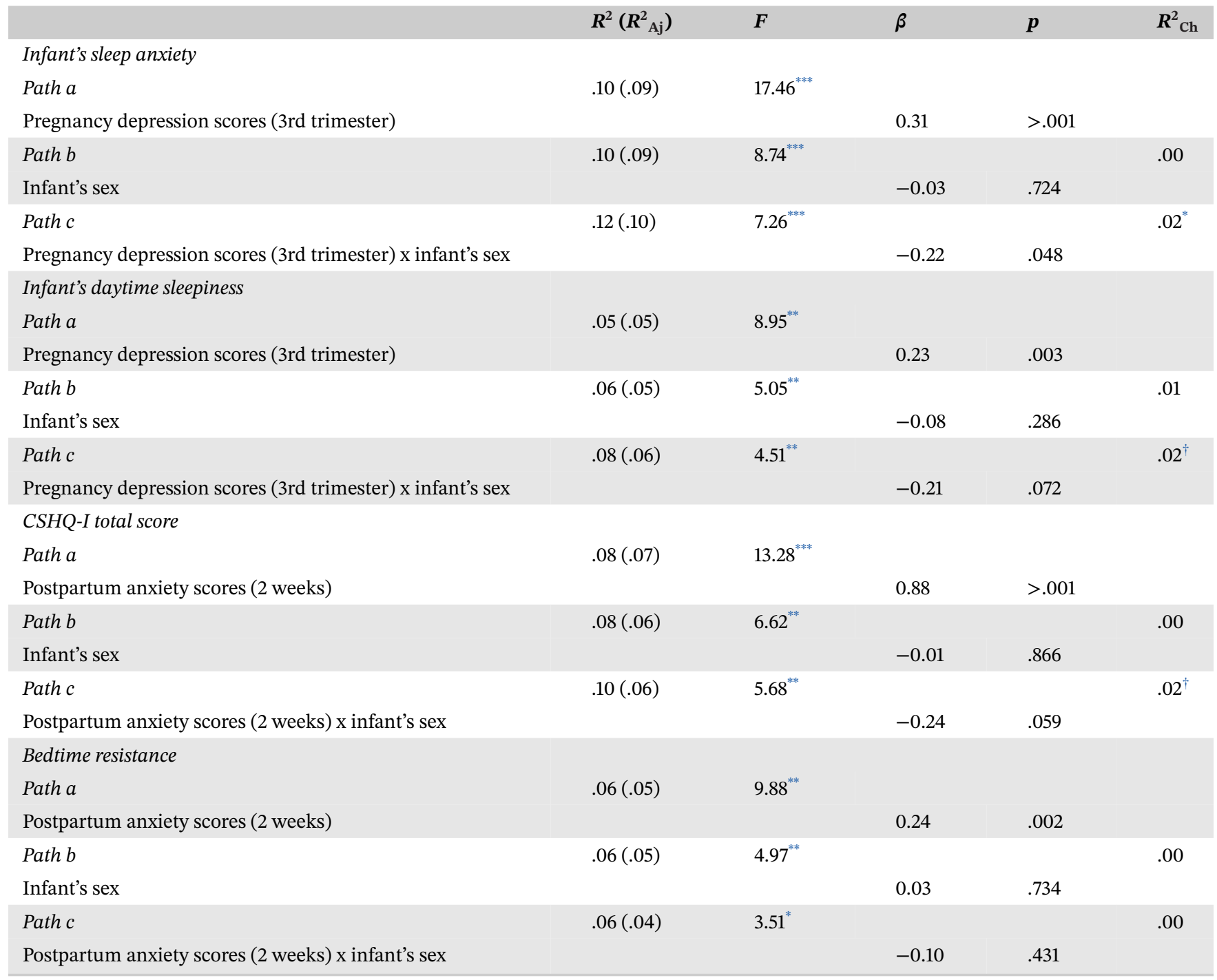

Note. $R_{\mathrm{Aj}}^{2}=$ adjusted $R^{2} ; R^{2}{ }_{\mathrm{Ch}}=R^{2}$ change.

${ }^{\dagger} p>0.05<0.10 .{ }^{*} p<.05 .{ }^{* *} p<.01 .{ }^{* * *} p<.001$.

problems may contribute to the emergence of mother's postpartum anxiety and depression symptoms (Ystrom, Nilsen, Hysing, Sivertsen, \& Ystrom, 2017), which may help to explain the association between mother's prenatal and postpartum depression.

Mother's postpartum depression symptoms at 2 weeks (EPDS total score) were found to predict more infant's sleep problems at 6 months, specifically more bedtime resistance and total sleep problems at 6 months. This result is in line with previous research associating mother's postpartum depression with infant's sleep problems (GressSmith et al., 2012; Hughes et al., 2015), specifically during the early postpartum period (Dennis \& Ross, 2005). The present study adds to this previous literature by showing that the effects of mother's postpartum depression symptoms on infant's sleep problems can occur specifically from mother's depression symptoms in the early weeks postpartum, a period where mothers often present poor sleep quality (Hunter, Rychnovsky, \& Yount, 2009). Poor maternal sleep quality has been associated with both mother's depression (Lawson, Murphy, Sloan, Uleryk, \& Dalfen, 2015; Park, Meltzer-Brody, \& Stickgold, 2013) and infant's sleep problems (Dennis \& Ross, 2005). When further analyses were performed with separated anxiety and depression scores of the EPDS, mother's anxiety scores at 2 weeks postpartum significantly predicted more bedtime resistance and total sleep problems at 6 months. These results suggest that the anxiety scores of the EPDS during the first weeks of the postpartum period have a major role on the emergence of further infant's sleep problems.

This study extended the knowledge of previous studies showing that mother's prenatal (Gerardin et al., 2011; 
Nevarez et al., 2010; O'Connor et al., 2007) and postpartum depression (Dennis \& Ross, 2005; Gress-Smith et al., 2012; Hughes et al., 2015) predict infant's sleep problems. Mother's prenatal and 2 weeks postpartum depression symptoms predict different specific sleep problems, suggesting that infant's sleep problems may be affected through different mechanisms. Additionally, while during pregnancy, depression scores of the EPDS predict infant's sleep problems, anxiety scores of the EPDS at 2 weeks postpartum were found to predict infant's sleep problems. These results are congruent and reinforce the literature that suggests that the effects of mother's depression on infant development may occur through different and interactive mechanisms (Glover et al., 2018; Stein et al., 2014). These results also suggest that these mechanisms may interact differently according to the timing of mother's depression symptoms. The genetic factors, epigenetic change, or dysfunctional biological mechanisms, resultant from mother's depression symptoms during pregnancy may predict the emergence of infant's sleep anxiety and daytime sleepiness. Conversely, infant's CSHQ-I total score and bedtime resistance may be associated with the maladaptive cognitions and interactions presented by the mothers during the early postpartum period, especially associated with mother's anxiety symptoms.

Infant's sex specific effects of mother's prenatal depression symptoms were also found. Boys of mothers with more depression symptoms at the third trimester of pregnancy presented more sleep anxiety at 6 months. These results are congruent with previous studies showing that boys are more vulnerable to the impact of mother's prenatal depression (Beeghly et al., 2017; McGinnis et al., 2015; Netsi et al., 2015). These results may also be interpreted within the framework of differential susceptibility, suggesting that boys and girls are not equally affected by an early risk factor (Belsky et al., 2007; Netsi et al., 2015). Nonsignificant interaction effects between mother's prenatal depression symptoms and infant's sex were shown on infant's daytime sleepiness and nonsignificant interaction effects between mother's depression symptoms at 2 weeks postpartum and infant's sex were found on infant's CSHQ-I total problems and bedtime resistance. These results suggest that infant's sex specific effects only occur in specific dimensions of infant's sleep problems, namely in sleep anxiety. Previous studies already shown that male fetuses present more difficulty in adapting to a prenatal adverse environment than females (Sutherland \& Brunwasser, 2018), and thus are at higher risk of presenting more anxiety related with sleep.

This study presents some strengths, namely by (a) analyzing the concurrent effect of both mother's prenatal and postpartum depression symptoms on infant's sleep prob- lems; (b) considering different specific infant's sleep problems; (c) clarifying the effect of infant's sex; and (d) presenting a longitudinal design, with a community sample followed since the third trimester of pregnancy to 6 months postpartum. Some limitations can also be identified. Both dependent and independent variables were assessed through the same informant, which may have led to an increase of the common shared variance. Mother's prenatal depression symptoms were only assessed at the third trimester of pregnancy, which did not account for any depression symptoms experienced during the first or second trimester. The generalization of this study's findings should be performed with caution, considering that primiparous women were more likely to complete all the study measures. However, women who completed all the study measures did not differ from those who did not complete all the study measures in any other sociodemographic variable. The small variance explained by the different tested models also suggests that other variables may affect infant's sleep problems. Additionally, the assessment of other variables that may also be involved in the emergence of infant's sleep problems was not included, namely infant's temperament, parenting quality, maternal anxiety symptoms or sleep quality.

\section{4 | IMPLICATIONS FOR RESEARCH AND CLINICAL PRACTICE}

This study highlighted the role of both mother's prenatal and postpartum depression symptoms on the emergence of infant's sleep problems. This finding presents relevant implications for both research and clinical practice, considering that sleep problems during infancy are one common concern for parents and clinicians and are usually predictors of further sleep, behavioral and emotional problems (Byars et al., 2012; Sivertsen et al., 2015; Williams, Berthelsen, Walker, \& Nicholson, 2017). Future research may explore the effect of other variables, namely infant's individual characteristics and maternal sleep quality and anxiety on the emergence of infant's sleep problems.

Regarding the implications for clinical practice, these findings show the importance of screening for mother's prenatal depression and depression in the early postpartum period as part of routine perinatal care to identify infants at risk of presenting developmental problems. Moreover, considering that mother's prenatal and postpartum depression symptoms both affect infant's sleep problems, these results acknowledge the need of intervening on infant's sleep problems when developing mother's depression interventions. This study also identified infant's sex specific effects. By showing that male's and female's sleep problems are differentially affected by maternal 
prenatal depression symptoms, this study highlights the importance of designing targeted interventions.

\section{5 | CONCLUSIONS}

This study provides a broader picture of the effects of mother's prenatal and postpartum depression symptoms on infant's sleep problems at 6 months. Mother's depression during pregnancy and the early postpartum period both predict more infant's sleep problems at 6 months. Additionally, boys seem more vulnerable to the effects of mother's prenatal depression symptoms. These results extend the knowledge on infant's sleep research, by showing (a) the effects of both mother's prenatal and postpartum depression symptoms on infant's sleep problems at 6 months, and (b) the sex dependent impact of mother's prenatal depression symptoms on infant's sleep anxiety.

\section{CONFLICT OF INTEREST}

The author declares that there is no conflict of interest that could be perceived as prejudicing the impartiality of the research reported.

\section{O R C I D}

Cláudia Castro Dias (D https://orcid.org/0000-0002-5511$457 \mathrm{X}$

\section{REFERENCES}

Baron, R. M., \& Kenny, D. A. (1986). The moderator-mediator variable distinction in social psychological research: Conceptual, strategic, and statistical considerations. Journal of Personality and Social Psychology, 51(6), 1173-1182.

Bayer, J. K., Hiscock, H., Hampton, A., \& Wake, M. (2007). Sleep problems in young infants and maternal mental and physical health. Journal of Paediatrics and Child Health, 43, 66-73. https://doi.org/ 10.1111/j.1440-1754.2007.01005.x

Beeghly, M., Partridge, T., Tronick, E., Muzik, M., Mashhadi, M. R., Boeve, J. L., \& Irwin, J. L. (2017). Associations between early maternal depressive symptom trajectories and toddlers' felt security at 18 months: Are boys and girls at differential risk? Infant Mental Health Journal, 38, 53-67. https://doi.org/10.1002/imhj.21617

Belsky, J., Bakermans-Kranenburg, M. J., \& Van I. Jzendoorn, M. H. (2007). For better and for worse: Differential susceptibility to environmental influences. Current Directions in Psychological Science, 16, 300-304. https://doi.org/10.1111/j.1467-8721.2007.00525.x

Byars, K. C., Yolton, K., Rausch, J., Lanphear, B., \& Beebe, D. W. (2012). Prevalence, patterns, and persistence of sleep problems in the first 3 years of life. Pediatrics, 129, e276-e284. https://doi.org/ 10.1542/peds.2011-0372

Couto, T. C., Cardoso, M. N., Brancaglion, M. M., Faria, G. C., Garcia, F. D., Nicolato, R., ... Corrêa, H. (2016). Antenatal depression: Prevalence and risk factor patterns across the gestational period. Journal of Affective Disorders, 192, 70-75. https://doi.org/10.1016/j. jad.2015.12.017
Cox, J. L., Holden, J. M., \& Sagovsky, R. (1987). Detection of postnatal depression: Development of the 10-item Edinburgh Postnatal Depression Scale. British Journal of Psychiatry, 150, 782-786. https://doi.org/10.1192/bjp.150.6.782

Dennis, C. L., \& Ross, L. (2005). Relationships among infant sleep patterns, maternal fatigue, and development of depressive symptomatology. Birth, 32, 187-193. https://doi.org/10.1111/j.0730-7659. 2005.00368.x

Dias, C. C., Figueiredo, B., \& Pinto, T. M. (2018). Children's Sleep Habits Questionnaire-infant version. Jornal de Pediatria (Rio J), 94, 146-152. https://doi.org/10.1016/j.jped.2017.05.012.

DiPietro, J. A., \& Voegtline, K. M. (2017). The gestational foundation of sex differences in development and vulnerability. Neuroscience, 342, 4-20. https://doi.org/10.1016/j.neuroscience.2015.07.068

Field, T. (2010). Postpartum depression effects on early interactions, parenting, and safety practices: A review. Infant Behavior \& Development, 33(1), 1-6. https://doi.org/10.1016/j.infbeh.2009.10.005

Field, T., Diego, M., Hernandez-Reif, M., Figueiredo, B., Schanberg, S., \& Kuhn, C. (2007). Sleep disturbances in depressed pregnant women and their newborns. Infant Behavior \& Development, 30, 127-133. https://doi.org/10.1016/j.infbeh.2006.08.002

Figueiredo, B., \& Conde, A. (2011a). Anxiety and depression in women and men from early pregnancy to 3-months postpartum. Archives of Women's Mental Health, 14, 247-255. https://doi.org/10. 1007/s00737-011-0217-3

Figueiredo, B., \& Conde, A. (2011b). Anxiety and depression symptoms in women and men from early pregnancy to 3-months postpartum: Parity differences and effects. Journal of Affective Disorders, 132, 146-157. https://doi.org/10.1016/j.jad.2011.02.007

Figueiredo, F., Dias, C. C., Pinto, T., \& Field, T. (2016). Infant sleepwake behaviors at two weeks, three and six months. Infant Behavior and Development, 44, 169-178. https://doi.org/10.1016/j.infbeh. 2016.06.011

Figueiredo, B., Pinto, T. M., Pacheco, A., \& Field, T. (2017). Fetal heart rate variability mediates prenatal depression effects on neonatal neurobehavioral maturity. Biological Psychology, 123, 294-301. https://doi.org/10.1016/j.biopsycho.2016.10.013

Galland, B. C., Taylor, B. J., Elder, D. E., \& Herbison, P. (2012). Normal sleep patterns in infants and children: A systematic review of observational studies. Sleep Medicine Reviews, 16, 213-222. https: //doi.org/10.1016/j.smrv.2011.06.00

Gentile, S. (2017). Untreated depression during pregnancy: Short-and long-term effects in offspring. A systematic review. Neuroscience, 342, 154-166. https://doi.org/10.1016/j.neuroscience.2015.09.001

Gerardin, P., Wendland, J., Bodeau, N., Galin, A., Bialobos, S., Tordjman, S., ... Cohen, D. (2011). Depression during pregnancy: Is the developmental impact earlier in boys? A prospective case-control study. Journal of Clinical Psychiatry, 72, 378-387. https://doi.org/ 10.4088/JCP.09m05724blu

Glover, V., O’Donnell, K. J., O’Connor, T. G., \& Fisher, J. (2018). Prenatal maternal stress, fetal programming, and mechanisms underlying later psychopathology-A global perspective. Development and Psychopathology, 30, 843-854. https://doi.org/10.1017/ S095457941800038X

Goodlin-Jones, B. L., Burnham, M. M., Gaylor, E. E., \& Anders, T. F. (2001). Night waking, sleep-wake organization, and self-soothing in the first year of life. Journal of Developmental \& Behavioral Pediatrics, 22, 226-233. https://doi.org/10.1097/00004703-20010800000003 
Goodlin-Jones, B. L., Sitnick, S. L., Tang, K., Liu, J., \& Anders, T. F. (2008). The Children's Sleep Habits Questionnaire in toddlers and preschool children. Journal of Developmental \& Behavioral Pediatrics, 29, 82-88. https://doi.org/10.1097/DBP.0b013e318163c39a

Gress-Smith, J. L., Luecken, L. J., Lemery-Chalfant, K., \& Howe, R. (2012). Postpartum depression prevalence and impact on infant health, weight, and sleep in low-income and ethnic minority women and infants. Maternal and Child Health Journal, 16(4), 887-893. https://doi.org/10.1007/s10995-011-0812-y

Hughes, A., Gallagher, S., \& Hannigan, A. (2015). A cluster analysis of reported sleeping patterns of 9-month old infants and the association with maternal health: Results from a population based cohort study. Maternal and Child Health Journal, 19, 1881-1889. https://doi.org/10.1007/s10995-015-1701-6

Hunter, L. P., Rychnovsky, J. D., \& Yount, S. M. (2009). A selective review of maternal sleep characteristics in the postpartum period. Journal of Obstetric, Gynecologic \& Neonatal Nursing, 38, 60-68. https://doi.org/10.1111/j.1552-6909.2008.00309.x

Jomeen, J., \& Martin, C. R. (2005). Confirmation of an occluded anxiety component within the Edinburgh Postnatal Depression Scale (EPDS) during early pregnancy. Journal of Reproductive and Infant Psychology, 23, 143-154, https://doi.org/10.1080/ 02646830500129297

Lawson, A., Murphy, K. E., Sloan, E., Uleryk, E., \& Dalfen, A. (2015). The relationship between sleep and postpartum mental disorders: A systematic review. Journal of Affective Disorders, 176, 65-77. https://doi.org/10.1016/j.jad.2015.01.017

Matthey, S. (2008). Using the Edinburgh Postnatal Depression Scale to screen for anxiety disorders. Depression and Anxiety, 25, 926931. https://doi.org/10.1002/da.20415

McGinnis, E., Bocknek, E., Beeghly, M., Rosenblum, K. L., \& Muzik, M. (2015). Does child sex moderate vulnerability to postpartum risk among infants of mothers at risk for psychopathology? Infancy, 20, 42-69. https://doi.org/10.1111/infa.12065

Netsi, E., van IJzendoorn, M. H., Bakermans-Kranenburg, M. J., Wulff, K., Jansen, P. W., Jaddoe, V. W., ... Ramchandani, P. G. (2015). Does infant reactivity moderate the association between antenatal maternal depression and infant sleep?. Journal of Developmental and Behavioral Pediatrics: JDBP, 36, 440-449. https:// doi.org/10.1097/DBP.0000000000000181.

Nevarez, M. D., Rifas-Shiman, S. L., Kleinman, K. P., Gillman, M. W., \& Taveras, E. M. (2010). Associations of early life risk factors with infant sleep duration. Academic Pediatrics, 10, 187-193. https://doi. org/10.1016/j.acap.2010.01.007

O’Connor, T. G., Caprariello, P., Blackmore, E. R., Gregory, A. M., Glover, V., \& Fleming, P. \& ALSPAC Study Team.(2007) Prenatal mood disturbance predicts sleep problems in infancy and toddlerhood. Early Human Development, 83, 451-458. https://doi.org/10. 1016/j.earlhumdev.2006.08.006

Owens, J. A., Spirito, A., \& McGuinn, M. (2000). The Children's Sleep Habits Questionnaire (CSHQ): Psychometric properties of a survey instrument for school-aged children. Sleep, 23, 1043-1052. https://doi.org/10.1093/sleep/23.8.1d

Park, E. M., Meltzer-Brody, S., \& Stickgold, R. (2013). Poor sleep maintenance and subjective sleep quality are associated with postpartum maternal depression symptom severity. Archives of Women's Mental Health, 16, 539-547. https://doi.org/10.1007/ s00737-013-0356-9
Phillips, J., Charles, M., Sharpe, L., \& Matthey, S. (2009). Validation of the subscales of the Edinburgh Postnatal Depression Scale in a sample of women with unsettled infants. Journal of Affective Disorders, 118, 101-112. https://doi.org/10.1016/j.jad.2009.02.004

Rosenfeld, C. S. (2015). Sex-specific placental responses in fetal development. Endocrinology, 156, 3422-3434. https://doi.org/10.1210/ en.2015-1227

Rutter, M. (1996). Transitions and turning points in developmental psychopathology: As applied to the age span between childhood and mid-adulthood. International Journal of Behavioral Development, 19(3), 603-626.

Rutter, M., \& Sroufe, L. A. (2000). Developmental psychopathology: Concepts and challenges. Development and Psychopathology, 12, 265-296. https://doi.org/10.1017/S0954579400003023

Sandman, C., Glynn, L., \& Davis, E. (2013). Is there a viabilityvulnerability tradeoff? Sex differences in fetal programming. Journal of Psychosomatic Research, 75, 327-335. https://doi.org/10.1016/ j.jpsychores.2013.07.009

Sette, S., Baumgartner, E., Ferri, R., \& Bruni, O. (2017). Predictors of sleep disturbances in the first year of life: A longitudinal study. Sleep Medicine, 36, 78-85. https://doi.org/10.1016/j.sleep.2017.04. 015

Sivertsen, B., Harvey, A. G., Reichborn-Kjennerud, T., Torgersen, L., Ystrom, E., \& Hysing, M. (2015). Later emotional and behavioral problems associated with sleep problems in toddlers: A longitudinal study. JAMA Pediatrics, 169, 575-582. https://doi.org/10.1001/ jamapediatrics.2015.0187

Smith-Nielsen, J., Matthey, S., Lange, T., \& Væver, M. S. (2018). Validation of the Edinburgh Postnatal Depression Scale against both DSM-5 and ICD-10 diagnostic criteria for depression. BMC Psychiatry, 18, 393-405. https://doi.org/10.1186/s12888-018-1965-7

Sroufe, L. A. (2009). The concept of development in developmental psychopathology. Child Development Perspectives, 3, 178-183. https: //doi.org/10.1111/j.1750-8606.2009.00103.x

Sroufe, L. A. (2016). Pathways to adaptation and maladaptation: Psychopathology as developmental deviation. In D. Cicchetti (Ed.), The emergence of a discipline: Rochester symposium on developmental psychopathology (Vol. 1, pp. 27-54). Vol.New York, NY: Taylor \& Francis Group.

Stein, A., Pearson, R. M., Goodman, S. H., Rapa, E., Rahman, A., McCallum, M., ... Pariante, C. M. (2014). Effects of perinatal mental disorders on the fetus and child. The Lancet, 384, 1800-1819. https://doi.org/10.1016/S0140-6736(14)61277-0

Sutherland, S., \& Brunwasser, S. M. (2018). Sex differences in vulnerability to prenatal stress: A review of the recent literature. Current Psychiatry Reports, 20, 102. https://doi.org/10.1007/s11920018-0961-4

Tendais, I., Costa, R., Conde, A., \& Figueiredo, B. (2014). Screening for depression and anxiety disorders from pregnancy to postpartum with the EPDS and STAI. The Spanish Journal of Psychology, 17, 1-9. https://doi.org/10.1017/sjp.2014.7.

Underwood, L., Waldie, K., D’Souza, S., Peterson, E. R., \& Morton, S. (2016). A review of longitudinal studies on antenatal and postnatal depression. Archives of Women's Mental Health, 19, 711-720. https: //doi.org/10.1007/s00737-016-0629-1

Verreault, N., Da Costa, D., Marchand, A., Ireland, K., Dritsa, M., \& Khalifé, S. (2014). Rates and risk factors associated with depressive symptoms during pregnancy and with postpartum onset. Journal 
of Psychosomatic Obstetrics \& Gynecology, 35, 84-91. https://doi. org/10.3109/0167482X.2014.947953

Waumans, R. C., Terwee, C. B., Van den Berg, G., Knol, D. L., Van Litsenburg, R. R., \& Gemke, R. J. (2010). Sleep and sleep disturbance in children: Reliability and validity of the Dutch version of the Child Sleep Habits Questionnaire. Sleep, 33, 841-845. https: //doi.org/10.1093/sleep/33.6.841

Williams, K. E., Berthelsen, D., Walker, S., \& Nicholson, J. M. (2017). A developmental cascade model of behavioral sleep problems and emotional and attentional self-regulation across early childhood. Behavioral Sleep Medicine, 15(1), 1-21. https://doi.org/10. 1080/15402002.2015.1065410
Ystrom, H., Nilsen, W., Hysing, M., Sivertsen, B., \& Ystrom, E. (2017). Sleep problems in preschoolers and maternal depressive symptoms: An evaluation of mother-and child-driven effects. Developmental Psychology, 53, 2261. https://doi.org/10.1037/dev0000402

How to cite this article: Dias CC, Figueiredo B. Mother's prenatal and postpartum depression symptoms and infant's sleep problems at 6 months. Infant Ment Health J. 2020;1-14.

https://doi.org/10.1002/imhj.21869 